\title{
Acoustic absorption by the soft modes of defects in $\mathrm{NH}_{4} \mathrm{Cl}$
}

\author{
F. J. Bartis \\ Department of Physics, Indiana University, Bloomington, Indiana 47401, U.S.A.
}

(Reçu le 23 août 1979, révisé le 7 janvier, accepté le 8 février 1980)

\begin{abstract}
Résumé. - Des défauts intrinsèques tendent à osciller par rapport aux domaines dans la région diphasée de la transformation d'orientation. Traitant le solide comme un continu élastique, on trouve que les fréquences de vibrations des lacunes et des dislocations mobiles diminuent comme $\left(T_{0}-T\right)^{1 / 3}$ et $\left|T_{0}-T\right|^{1 / 2}$, respectivement, près du point de transition $T_{0}$. De l'excitation ultrasonore du mode mou de dislocations à pulsation $\omega$ le frottement intérieur acquiert des maximums déplacés à $T_{0} \pm k \omega^{2}$ et des queues étendues de la forme $\omega\left|T_{0}-T\right|^{-1}$. On propose une étude spectroscopique de l'opalescence de transition pour vérifier le devis de $50 \mathrm{MHz}$ comme une limite inférieure de la fréquence du mode mou de dislocations dans un bon cristal.
\end{abstract}

\begin{abstract}
Intrinsic defects tend to oscillate relative to the domains in the two-phase region of the orientational transformation. When the solid is treated as a continuous elastic medium, the vibrational frequencies of vacancies and mobile dislocations are found to decrease as $\left(T_{0}-T\right)^{1 / 3}$ and $\left|T_{0}-T\right|^{1 / 2}$, respectively, near the transition point $T_{0}$. From the ultrasonic excitation of the soft mode of dislocations at angular frequency $\omega$ the internal friction acquires displaced maxima at $T_{0} \pm k \omega^{2}$ and extended tails of the form $\omega\left|T_{0}-T\right|^{-1}$. A spectroscopic study of the transitional opalescence is proposed to check the estimate of $50 \mathrm{MHz}$ as a lower bound on the frequency of the soft mode of dislocations in a good crystal.
\end{abstract}

1. Introduction. - Valuable insight into the quasistatic properties of solids during weakly first-order transitions is provided by the continuum theory of lattice defects. By way of illustration consider the opalescence of ammonium chloride during its orientational ordering reaction. Assume that the light undergoes multiple Rayleigh-Gans scattering from the domains formed in the two-phase region of the reaction [1]. It can be plausibly argued that the intensity of the light emerging at $90^{\circ}$ is proportional to the surface area of the domains [2]. Suppose further that the transition points of the crystallites around a vacancy are determined solely by its $A / r^{3}$ stress field [3]. The transition curve of the reaction being almost a straight line, the radius of the spherical domain that develops around the vacancy should vary roughly as $\left(T_{0}-T\right)^{-1 / 3}$. In contrast, the radius of the cylindrical domains that appear alongside an edge dislocation should go approximately as $\left|T_{0}-T\right|^{-1}$, if its $B / R$ stress field [4] regulates domain development. Accordingly the intensity of the opalescence should diverge as $\left(T_{0}-T\right)^{-2 / 3}$ and/or $\left|T_{0}-T\right|^{-1}$. From the observation of both kinds of singular behaviour [5] it is concluded that domain evolution is controlled by vacancies in the lower part of the coexistence region and by dislocations in its upper portion [6].
The continuum theory of crystal imperfections also helps to explain the growing sluggishness of weakly first-order transformations near their nominal transition temperatures. In the spirit of irreversible thermodynamics let the speed of the domain boundary under isothermal conditions be proportional to the underpressure of the boundary. When the departure from equilibrium is minuscule, the underpressure becomes proportional to the gradient of the stress field of the vacancy or the dislocation at the domain boundary. It follows then that the thermal relaxation time diverges as $\left(T_{0}-T\right)^{-4 / 3}$ and/or $\left|T_{0}-T\right|^{-2}$. Again both types of divergent behaviour are seen in the equilibration of $\mathrm{Cu}_{3} \mathrm{Au}$ during its spatial ordering reaction [7].

2. Defect modes. - The vibrations of crystal imperfections in single-phase solids have been under active investigation for over thirty years [8]. Yet no thought seems to have been given to their vibrations in twophase solids. Here I want to show that intrinsic defects acquire special modes of oscillation during passage through coherent two-phase fields. Assuming that domain growth is regulated by the stresses around the defects, I determine that the oscillation frequencies of vacancies and mobile dislocations fall as $\left(T_{0}-T\right)^{1 / 3}$ and $\left|T_{0}-T\right|^{1 / 2}$, respectively, in the vicinity of the 
transition point. I suggest that these soft modes are primarily responsible for the dynamic anomalies of weakly first-order transformations.

Let me begin with a vacancy surrounded by a domain of the disordered modification of ammonium chloride. I presume that at the prevailing temperature the vacancy makes a large number of jumps per unit time between locally equivalent lattice sites. The domain boundary however moves much too slowly to keep up with the nomadic vacancy. Consequently the vacancy needs more energy in order to wander further from the domain centre. If its displacement $S$ from the centre is very small compared to the domain radius $r_{\mathrm{n}}$, then the vacancy must make up for the extra lattice strain energy

$$
\Delta E \approx 3 \pi f A S^{2} / r_{\mathrm{n}}^{2},
$$

where $f$ is the fractional change of volume in passing from the disordered to the partially ordered modification. Differentiating with respect to $S$ gives the force acting to return the vacancy to the centre of the domain

$$
C \approx 6 \pi f A S / r_{\mathrm{n}}^{2} .
$$

Subject to this force the vacancy tends to oscillate with an angular frequency $\omega_{\mathrm{v}}$ given by

$$
\omega_{\mathrm{v}}^{2} \approx 6 \pi f A / M r_{\mathrm{n}}^{2},
$$

where $M$ is the effective mass of the vacancy. With the insertion of the temperature variation of the radius it is seen that the oscillation frequency of the vacancy falls as $\left(T_{0}-T\right)^{1 / 3}$.

A similar line of reasoning can be used to treat the soft mode of edge dislocations. One envisages a shift of the whole dislocation line a distance $s$ to a locally equivalent lattice position. One assumes that under the circumstances the Peierls force on the mobile dislocation can be neglected. From the change in the lattice strain energy the restoring force per unit length of the dislocation is estimated to be [9]

$$
D \approx G b f s / \pi R_{\mathrm{n}},
$$

where $G$ is the shear modulus, $b$ is the length of the Burgers vector of the dislocation and $R_{\mathrm{n}}$ is the radius of the domain decorating the dislocation. Taking the effective mass per unit length to be $\pi \rho b^{2}$, one arrives at the expression

$$
\omega_{\mathrm{e}}^{2} \approx G f / \pi^{2} \rho b R_{\mathrm{n}} .
$$

Here $\rho$ is the density of ammonium chloride. Remembering that the radius goes roughly as $\left|T_{0}-T\right|^{-1}$, one concludes that $\omega_{\mathrm{e}}$ decreases as $\left|T_{0}-T\right|^{1 / 2}$.

The soft mode of edge dislocations should be carefully distinguished from their Koehler mode $[10,11]$. When the Koehler mode is excited, a dislocation pinned at several points along its length vibrates like a violin string. On the other hand, when the soft mode is excited, the whole length of a mobile dislocation oscillates back and forth in a slip plane. The frequency of the Koehler mode can be raised by shortening the length of the vibrating segments through, say, gamma irradiation of the crystal [12]. Likewise the frequency of the soft mode can be shifted by varying the size of the domain, lying alongside the dislocation, through a change in the temperature of the sample. There is in any case a lower bound set on the frequency of the soft mode by the defect density. It is a direct result of the limit on the domain size. In an amonium chloride crystal containing $4 \times 10^{4}$ dislocations $/ \mathrm{cm}^{2}$ the domain radius reaches about $10^{-2} \mathrm{~cm}$. From eq. (5) it follows that the soft mode of dislocations can only drop to $50 \mathrm{MHz}$ !

3. Ultrasonic losses. - I submit that resonant absorption by the soft mode of dislocations underlies the anomalous attenuation of longitudinal ultrasound in ammonium chloride $[13,14]$. Like the Koehler mode, the soft mode should be broadened by a strong radiation damping. In the formal description of the soft mode a retarding force varying as the speed of the dislocation can be used. The internal friction $Q^{-1}$ of such a damped resonance may be written [15]

$$
Q^{-2}=\frac{\omega^{2} \gamma^{2}}{\left(\omega_{\mathrm{e}}^{2}-\omega^{2}\right)^{2}+\omega^{2} \gamma^{2}},
$$

where $\gamma$ characterizes the damping force per unit mass per unit speed of the dislocation. It is $\omega_{\mathrm{e}}$ that causes the internal friction to vary during the orientational transformation. The internal friction passes through maxima when $\omega_{\mathrm{e}}=\omega$ or when the temperature is

$$
T_{\mathrm{m}}=T_{0} \pm k \omega^{2} \text {, }
$$

where $k$ is practically a constant. On the other hand, when $\omega_{\mathrm{e}} \gg \omega$, the temperature differs substantially from $T_{0}$ and the internal friction goes inversely as the temperature displacement

$$
Q^{-1} \approx \omega \gamma \omega_{\mathrm{e}}^{-2} \propto \omega \gamma\left|T_{0}-T\right|^{-1} .
$$

The soft mode of vacancies should also be subject to a strong radiation damping when it is excited acoustically. In this case the internal friction should exhibit a peak at

$$
T_{\mathrm{p}}=T_{0}-h \omega^{3}
$$

and a tail of the form

$$
Q^{-1} \approx \omega \delta \omega_{\mathrm{v}}^{-2} \propto \omega \delta\left(T_{0}-T\right)^{-2 / 3},
$$

where $h$ denotes a positive constant and $\delta$ characterizes the damping force per unit mass per unit speed of the vacancy.

Physically the long tails of the transitional internal friction indicate that the second modification of ammonium chloride is present, even when the temperature is far from $T_{0}$. The extended tails of its transitional opalescence may be viewed in a similar fashion. 
It may be recalled that the amount of light scattered by the domains lying alongside dislocation is proportional to their surface area and hence their radius. From eqs. (5) and (8) it can be inferred that the contribution of these domains to the internal friction is also proportional to their radius. For ten degrees Kelvin or so above $T_{0}$ the data on both parameters $[5,16,17]$ bear out such a relationship, if in line with the continuum description of lattice imperfections the radius is taken to be inversely proportional to the temperature displacement. It would be unrealistic to expect agreement over a larger temperature range, if a neutron diffraction study of the disordered state of manganous oxide is any guide. The weakly first-order nature of its antiferromagnetic transition near $122 \mathrm{~K}$ is clearly established by Bloch and Maury [18]. Renninger et al. [19] detect split peaks near the superlattice positions at 133 and $298 \mathrm{~K}$. They attribute the peaks to neutrons scattered by the antiferromagnetic modification of $\mathrm{MnO}$. However, they deduce from their data domain sizes of $46 \pm 5 \AA$ and $61 \pm 10 \AA$ at 133 and $298 \mathrm{~K}$, respectively. The nearly constant size probably means that in this temperature interval the second modification is almost all located near inclusions of $\mathrm{Mn}_{3} \mathrm{O}_{4}$ [20]. Returning to the orientational transformation of ammonium chloride, one notes that the growth of domains of its second modification near pinned dislocations would alter the frequency of the Koehler mode. Since the Koehler mode does not mimic the soft mode, the contribution of the pinned dislocations to the transitional internal friction would not follow eq. (8).

Blocked from the use of eqs. (8) and (10), one turns to eqs. (7) and (9) as a means of testing the present approach to ultrasonic absorption. Their predictions clearly differ from the implication of the Landau theory that the displacement of the peak varies as the first power of the frequency [21]. It should be emphasized that the displaced maxima of the internal friction are observable only if the acoustic frequency exceeds the lower bounds of the soft modes. These bounds can be estimated theoretically but it seems preferable to determine them experimentally. The experimental results can then serve as a check on the earlier calculation of $50 \mathrm{MHz}$ for a crystal having $4 \times 10^{4}$ dislocations $/ \mathrm{cm}^{2}$.

For this purpose I propose a measurement of the spectral width of the light quasielastically scattered by ammonium chloride during its orientational ordering reaction. Near $T_{0}$ the dynamic width is expected to remain finite, reflecting the boundedness of the defect modes. Lazay [5] finds that the opalescence of an ammonium chloride crystal can rise an order of magnitude when it is cycled through the reaction. In contrast, Yagi et al. [22] and Courtens [23] report that long anneals can greatly reduce the opalescence of potassium trihydrogen selenite and potassium dihydrogen phosphate. Extending the ideas of Cour- tens, I would argue that the passage of an ammonium chloride crystal through the reaction increases the density of intrinsic defects and so should raise the limiting frequencies of their soft modes. Thus I predict that an increase in the intensity of the Rayleigh or elastic component should be accompanied by a broadening of the dynamic component of the quasielastically scattered light.

4. Some questions. - The present letter is addressed to ammonium chloride during its orientational ordering reaction. Yet its findings are expected to apply to any solid undergoing a weakly first-order transition. This includes quartz during its $\alpha-\beta$ inversion. As the temperature of a quartz crystal is lowered toward the inversion point $T_{0}$, a normal mode of the lattice is observed to soften. According to Axe and Shirane [24] the frequency of this lattice mode decreases roughly as $\left(T-T_{\mathrm{c}}\right)^{1 / 2}$, where $T_{\mathrm{c}}$ is some ten degrees Kelvin below $T_{0}$. This lattice mode is therefore not the soft mode of mobile dislocations whose frequency is predicted to drop as $\left(T-T_{0}\right)^{1 / 2}$. Still the two modes may be related. Both seem to be governed by the same power-law exponent. On the other hand, a power-law exponent of $1 / 3$ appears to characterize the soft mode of the quartz lattice below the inversion point [25] as well as the soft mode of vacancies. Is there some fundamental reason for such an identity?

A lower bound of $50 \mathrm{MHz}$ is given here for the oscillation frequency of mobile dislocations in ammonium chloride during its orientational transformation. A similar limit should exist for the soft mode of dislocations in quartz. Such a limit would explain the failure of Shigenari et al. [26] to observe a dynamic component in the transitional opalescence of quartz between $1 \mathrm{~Hz}$ and $50 \mathrm{MHz}$. Studies like that of Shigenari et al. should be especially useful in deciding whether thermodynamic fluctuations play a major role in weakly first-order transitions. In the case of ammonium chloride a careful search for a dynamic component below $50 \mathrm{MHz}$ in its transitional opalescence could settle once and for all the question of whether critical fluctuations are responsible for its anomalous absorption of sound [27] and its strong scattering of light [5]. According to Ginzburg [28] and Benedek [29] the hallmark of critical opalescence is a spectral narrowing near the transition point. If the dynamic component does not go below $50 \mathrm{MHz}$, then by this criterion the phenomenon is not critical opalescence. Prevailing opinion seems ready to accept such a judgment for the $\alpha-\beta$ inversion of quartz [26] and the ferroelectric transformation of potassium dihydrogen phosphate [30] but not for the ferroelectric transition of lead germanate [31]. Is a two-phase region in the latter really ruled out by the experimental information at hand ? Could not its anomalous ultrasonic absorption [32] result from the excitation of the soft mode of dislocations? 


\section{References}

[1] Dinichert, P., Helv. Phys. Acta 15 (1942) 462.

[2] Bartis, F. J., J. Phys. C 6 (1973) L295.

[3] VAN Bueren, H. G., Imperfections in crystals (North-Holland, Amsterdam) 1960, p. 31.

[4] Ibid., p. 55.

[5] Lazay, P. D., Ph.D. Thesis, Massachusetts Institute of Technology (1969).

[6] Bartis, F. J., Phys. Lett. A 43 (1973) 61.

[7] BarTis, F. J., Acta Metall. 26 (1978) 879.

[8] LifShitZ, I. M., Adv. Phys. 13 (1964) 483.

[9] BarTis, F. J., J. Physique Lett. 40 (1979) L-351.

[10] KOEHLER, J. S., Imperfections in nearly perfect crystals, edited by W. Shockley, J. H. Hollomon, R. Maurer, F. Seitz (Wiley, New York) 1952, p. 197.

[11] Granato, A., Lücke, K., J. Appl. Phys. 27 (1956) 583.

[12] Stern, R. M., Granato, A. V., Acta Metall. 10 (1962) 358

[13] Garland, C. W., Jones, J. S., J. Chem. Phys. 42 (1965) 4194.

[14] Shustin, O. A., Yakovlev, I. A., Velichkina, T. S., JeTP Lett. 5 (1967) 3.

[15] See, for instance, reference [3], p. 357.

[16] Garland, C. W., Yarnell, C. F., J. Chem. Phys. 44 (1966) 3678.

[17] Leung, R. C., Zahradnik, C., Garland, C. W., Phys. Rev. $B 19$ (1979) 2612.
[18] Bloch, D., MaUry, R., Phys. Rev. B 7 (1973) 4883.

[19] Renninger, A., Moss, S. C., Averbach, B. L., Phys. Rev. 147 (1966) 418.

[20] Barber, D. J., Evans, R. G., J. Mater. Sci. 6 (1971) 1237.

[21] Belov, K. P., Katayev, G. I., Levitin, R. Z., J. Appl. Phys. 31 (1960) 153S.

[22] Yagi, T., Tanaka, H., Tatsuzaki, I., Phys. Rev. Lett. 38 (1977) 609.

[23] Courtens, E., Phys. Rev. Lett. 41 (1978) 1171.

[24] Axe, J. D., Shirane, G., Phys. Rev. B 1 (1970) 342.

[25] Scotr, J. F., Phys. Rev. Lett. 21 (1968) 907.

[26] Shigenari, T., Limura, Y., Takagi, Y., Opt. Commun. 31 (1979) 57.

[27] Matsushita, M., Phys. Rev. B 20 (1979) 1119.

[28] Ginzburg, V. L., Sov. Phys. Usp. 5 (1963) 649.

[29] BENEDEK, G. B., Light scattering spectra of solids, edited by G. B. Wright (Springer-Verlag, New York) 1969, p. 637.

[30] Mermelstein, M. D., Cummins, H. Z., Phys. Rev. B 16 (1977) 2177.

[31] Lyons, K. B., Fleury, P. A., Phys. Rev. B 17 (1978) 2403.

[32] Maishcik, E. P., Strukov, B. A., Sinyakov, E. V., Minaeva, K. A., MonyA, V. G., Sov. Phys. Solid State 19 (1977) 193. 